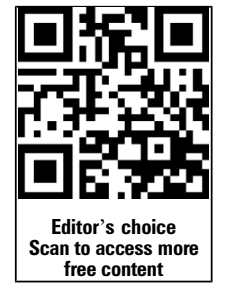
published online only. To view please visit the journal online (http://dx.doi.org/10.1136/ jmedgenet-2013-101693).

${ }^{1}$ Centre for Rare Diseases and Personalised Medicine, School of Clinical and Experimental Medicine, College of Medical and Dental Sciences, University of Birmingham, Birmingham, UK

${ }^{2}$ Department of Endocrinology, Birmingham Children's Hospital, Birmingham, UK ${ }^{3}$ Wellcome Trust Clinical Research Facility, University Hospitals Birmingham NHS Foundation Trust, Queen Elizabeth Hospital,

Birmingham, UK

${ }^{4}$ West Midlands Regional Genetics Service, Birmingham Women's Hospital, Birmingham, UK

${ }^{5}$ Department of Clinical Genetics, Northampton General Hospital NHS Trust, Northampton, UK

Correspondence to Dr Renuka P Dias, Centre for Rare Diseases and Personalised Medicine, School of Clinical and Experimental Medicine, College of Medical and Dental Sciences, University of Birmingham, Edgbaston, Birmingham B15 2TT, UK; r.dias.1@bham.ac.uk or Professor Eamonn R Maher Department of Medical Genetics, University of Cambridge, Box 238 Addenbrooke's Treatment Centre, Cambridge Biomedical Campus, Cambridge CB2 0Q0 UK; e.r.maher@bham.ac.uk

Received 28 March 2013 Revised 29 May 2013 Accepted 6 June 2013 Published Online First 28 June 2013

To cite: Dias RP, Nightingale P, Hardy C, et al. J Med Genet 2013;50:635-639.

\title{
Comparison of the clinical scoring systems in Silver-Russell syndrome and development of modified diagnostic criteria to guide molecular genetic testing
}

\author{
Renuka P Dias, ${ }^{1}, 2$ Peter Nightingale, ${ }^{3}$ Carol Hardy, ${ }^{4}$ Gail Kirby, ${ }^{1,4}$ Louise Tee, $^{1}$ \\ Susan Price, ${ }^{5}$ Fiona MacDonald, ${ }^{4}$ Timothy G Barrett, ${ }^{1,2}$ Eamonn R Maher ${ }^{1,4}$
}

\begin{abstract}
Background About half of all children with a clinical diagnosis of Silver-Russell syndrome (SRS) have a detectable molecular genetic abnormality (maternal uniparental disomy of chromosome upd(7)mat or hypomethylation of $\mathrm{H} 19$ differentially methylated region (DMR). The selection of children for molecular genetic testing can be difficult for non-specialists because of the broad phenotypic spectrum of SRS and the tendency of the facial features to mitigate during late childhood. Several clinical scoring systems for SRS have been developed by specialist researchers, but the utility of these for guiding molecular genetic testing in routine clinical practice has not been established.
\end{abstract}

Objectives To evaluate the utility of four published clinical scoring systems for genetic testing in a cohort of patients referred to a clinical service laboratory.

Patients Individuals with suspected SRS referred for molecular genetic testing of H19 DMR methylation status or upd(7)mat.

Results 36 of 139 (25.9\%) patients referred for testing had a genetic abnormality identified. Comparison of four published clinical scoring systems demonstrated that all included subjective criteria that could be difficult for the general clinician to assess. We developed a novel, simplified, scoring system utilising four objective, easily measured parameters that performed similarly to the most sensitive and specific published scoring system. Discussion Effective utilisation of genetic testing by clinicians without specialist clinical genetics training will be facilitated by the development of targeted testing protocols that are based on robust objective clinical features and are designed for use in a busy clinical practice rather than a research setting.

\section{INTRODUCTION}

Silver-Russell syndrome (SRS; OMIM 180860) is a clinically and genetically heterogeneous condition characterised by low birthweight, variable poor postnatal growth, and a number of dysmorphic features including a distinctive triangular facies, relative macrocephaly, and limb/facial asymmetry. The incidence of the syndrome is estimated between 1 in 3000 to 1 in 100000 depending on the stringency of criteria used. ${ }^{1}$ Approximately $10 \%$ of children with SRS are known to have maternal uniparental disomy of chromosome 7 (upd(7)mat). ${ }^{2}$ A further $40-50 \%$ will have hypomethylation of
H19 differentially methylated region (DMR) at the $11 \mathrm{p} 15$ locus. $^{3}{ }^{4}$ However, up to $50 \%$ of children with clinical features of SRS will have no identified genetic abnormality.

The absence of agreed consensus diagnostic criteria and the wide phenotypic variability of secondary clinical features means that SRS is often considered as a potential diagnosis but only a fraction of potential cases are confirmed by molecular analysis. Although patients with H19 DMR hypomethylation are more likely to have a 'classical SRS phenotype' than those without a detectable abnormality, it is widely recognised that the selection of potential SRS cases for genetic testing can be challenging. ${ }^{4}$ Furthermore, it is well recognised that the facial dysmorphism tends to become less obvious during late childhood. Hence several research groups have suggested clinical feature scoring systems that can be used for the clinical diagnosis of SRS and/or selection of potential cases for genetic testing. ${ }^{1}{ }^{4-6}$ However, there are no universally agreed clinical scoring systems and those that have been developed have usually been based on the results of testing in a research laboratory, in patient cohorts that were ascertained for research studies, and assessed by clinicians with a special interest in SRS. For genetic testing to become an integral part of mainstream clinical medicine it is important that reliable guidelines for selecting those patients who will benefit from molecular testing are provided to non-specialist clinicians. We therefore decided to compare four SRS clinical feature scoring systems in a large cohort of potential SRS patients who were ascertained through referral for genetic testing for SRS in a clinical diagnostic laboratory. Following on from this analysis we developed a simplified four point clinical assessment scoring system, suitable for a broad range of specialist and non-specialist clinicians that we suggest will provide robust criteria for selecting potential SRS patients for molecular testing.

\section{METHODS}

\section{Patients}

We studied individuals with a clinically suspected diagnosis of SRS who were referred to the West Midlands Regional Genetics laboratory for NHS diagnostic testing for SRS between January 2002 and December 2012. All SRS referrals were logged 
into a database and the referring clinicians (clinical geneticists, general paediatricians, and paediatric endocrinologists from across the UK) were asked to complete a standardised clinical features form (see online supplementary data).

\section{Molecular genetic analysis}

Methylation analysis for H19 DMR hypomethylation

Patients were assessed for H19 DMR hypomethylation using either SALSA MS-MLPA kit ME-030 (MRC Holland, Amstersdam, Netherlands) or pyrosequencing (Qiagen, Crawley, UK) using standard protocols. ${ }^{78}$ In addition, testing for UPD11 was performed using microsatellite marker analysis (D11S2071, D11S4046, D11STH, D11S1318, and D11SHBB). All DNA samples were tested twice to confirm methylation levels.

\section{upd(7)mat analysis}

Patients were assessed for upd(7)mat using standard protocol for microsatellite analysis (six markers, three each on $7 \mathrm{q}$ and $7 \mathrm{p}$ ). A positive diagnosis required evidence of unique maternal inheritance at $\geq 2$ markers. ${ }^{9}$

\section{Clinical scoring}

Four clinical scoring systems have been published for SRS and are summarised in table 1 . Criteria underlined in the table are subjective or difficult to assess within a single examination. Of note, only the Netchine criteria have a mandatory clinical feature which is required for diagnosis (low birth weight/length SD scores $(\mathrm{SDS})<-2$ for gestational age). ${ }^{4}$ Patients without any clinical information on the database were excluded from further analysis.

\section{Statistical analysis}

Data were analysed using SPSS Statistical package (PASW Statistics 18; RD, PN). Logistic regression was used to determine the predictive power of different models and receiver operator curves (ROC) drawn to determine the accuracy of these different models (figure 1).

For all the patients referred for diagnostic testing of upd(7) mat and hypomethylation of H19 DMR in the database, with sufficient clinical information, those with and without an identified genetic abnormality were assessed using the four different scoring systems. Where criteria were missing, these patients were excluded from the analysis of sensitivity and specificity of the scoring system. Of note, very few patients had enough data available for full comparison of the Bartholdi scoring system, ${ }^{5}$ therefore sensitivity and specificity were calculated from the original article. For analyses, the presence of the required specified clinical features was considered a positive test result, and the result of the molecular genetics testing (upd(7)mat or H19 DMR hypomethylation) was considered to indicate the presence or absence of SRS. The caveat to this is that up to $50 \%$ of clinical cases will have a negative genetic test result.

\section{RESULTS}

\section{General demographic data}

Sufficient clinical information to evaluate the four clinical scoring systems was available for 139 (79 male, 60 female)/257 referrals.

The median age of patients referred for analysis was 3.25 (range 0.25-21 years).

Information on the method of conception was available for 106 referrals; 7/106 (6.6\%) patients had been conceived by assisted reproductive technologies (ART) (1/7 ART conceived patients had H19 DMR hypomethylation and 6/7 had no genetic abnormality identified). It is now generally accepted that there is an increased frequency of children conceived by ART (intracytoplasmic sperm injection or in vitro fertilisation) in cohorts of children with Beckwith-Wiedemann syndrome (BWS). ${ }^{10-13}$ However, the link between ART and SRS is less well established, possibly because, as with our cohort, the numbers are too small. ${ }^{14}$ Although the frequency of children conceived by ART referred for SRS testing was higher than the UK average $(5.0 \%$ vs $1.8 \%)$, this might have been ascertainment bias. ${ }^{15}$

\section{Identification of a genetic abnormality and clinical data}

Thirty-six of 139 patients (25.9\%) had an identified genetic abnormality: 11/36 (30.6\%) upd(7)mat; 25/36 (69.4\%) H19 DMR hypomethylation. There were no differences between mean age at genetic testing between those with H19 DMR hypomethylation, upd(7)mat and no genetic abnormality. Of those with H19 DMR hypomethylation, the mean methylation index was 20.3\% (range 36.3-8.5\%; SD 4.9\%).

There were two variables where there was a significant difference between the groups. Patients with H19 DMR hypomethylation had significantly lower birthweights than those with no abnormality (H19 DMR hypomethylation median birthweight SDS $=-2.85$ vs no abnormality median birthweight $=-1.77$ $(\mathrm{p}<0.05))$. In addition, these patients were also significantly more likely to have asymmetry compared to either those with upd(7)mat or no genetic abnormality identified (H19=81\%; upd(7)mat $=10 \%$ and no abnormality $=20 \% ; \mathrm{p}<0.001$ ) (see online supplementary table $\mathrm{S} 1$ ).

As can be seen in table 2, the scoring system devised by Lai et al gives the best sensitivity and specificity when used on our UK based cohort. The scoring system from Bartholdi et al gives the highest sensitivity (ie, true positive when compared to genetic testing) but includes many data that are either not recorded as standard in child health records (birth length, birth occipitofrontal circumference (OFC)) or are difficult to assess at single assessments in early life when the diagnosis is most likely to be raised (genital abnormalities, developmental delay). All four systems use at least one subjective variable (prominent forehead or typical facies) which can be difficult for the clinician to judge given the rarity of the condition. Therefore, we investigated how a novel simpler scoring system based on only a few objective criteria would compare to the more complicated scoring systems developed in a research setting. The 'Birmingham SRS screening score' required three out of the following four criteria to be present for referral for molecular diagnosis:

1. Low birthweight (birthweight SDS $<-2$ )

2. Poor postnatal growth (height $S D S<-2$ after 2 years of age)

3. Relative macrocephaly (head circumference SDS $>1.5$ than height SDS)

4. Limb/body asymmetry.

As can be seen from online supplementary table S2, patients with H19 DMR hypomethylation had increased risk of asymmetry and lower birthweight compared to patients with upd(7) mat (as previously reported). There is a significant difference between birthweight SDS in those patients with H19 DMR hypomethylation and patients with no genetic abnormality identified ( $p=0.016$, Tukey's honestly significant difference test). Furthermore there is a significant difference between the incidence of asymmetry in patients with H19 DMR hypomethylation and both upd(7)mat and without any genetic abnormality identified $(p=0.007$ and $p<0.001$, respectively, Fisher's exact test with Bonferroni correction). Additionally, the relative macrocephaly was more pronounced in those with H19 DMR 
Table 1 Summary of published scoring systems for diagnosis of Silver-Russell syndrome (SRS)

\begin{tabular}{|c|c|c|c|c|c|}
\hline $\begin{array}{l}\text { Scoring } \\
\text { system }\end{array}$ & 1: Lai et $a l^{6}$ & 2: Price et $a l^{1}$ & 3: Netchine et $a l^{4}$ & 4: Bartholdi et $a l^{5}$ & 5: Birmingham \\
\hline Testing context & $\begin{array}{l}\text { Before association of } \mathrm{H} 19 \\
\text { DMR hypomethylation } \\
\text { with SRS }\end{array}$ & $\begin{array}{l}\text { Before association of } \mathrm{H} 19 \\
\text { DMR hypomethylation } \\
\text { with SRS }\end{array}$ & $\begin{array}{l}\text { Predominantly used in } \\
\text { assessment of patients with } \\
\text { H19 DMR hypomethylation }\end{array}$ & $\begin{array}{l}\text { Used to assess patients with } \\
\text { upd(7)mat and H19 DMR } \\
\text { hypomethylation }\end{array}$ & $\begin{array}{l}\text { Non-research cohort } \\
\text { Used to assess patients with } \\
\text { upd(7)mat and } H 19 \mathrm{DMR} \\
\text { hypomethylation }\end{array}$ \\
\hline $\begin{array}{l}\text { Mandatory } \\
\text { clinical } \\
\text { features }\end{array}$ & Nil & Nil & $\begin{array}{l}\text { Prenatal growth retardation } \\
\text { (birth weight/length } S D S<-2 \text { ) }\end{array}$ & Nil & Nil \\
\hline $\begin{array}{l}\text { Total number } \\
\text { of features } \\
\text { assessed }\end{array}$ & $\begin{array}{l}5 \text { (low birth weight; } \\
\text { postnatal short stature; } \\
\text { distinctive facies; } \\
\text { asymmetry and } \\
\text { clinodactyly) }\end{array}$ & $\begin{array}{l}5 \text { (low birth weight; } \\
\text { postnatal short stature; } \\
\text { relative macrocephaly; } \\
\text { distinctive facies and } \\
\text { asymmetry) }\end{array}$ & $\begin{array}{l}6 \text { (short stature -height } \\
\text { SDS<-2 after } 2 \text { years; } \\
\text { relative macrocephaly; } \\
\text { prominent forehead; } \\
\text { asymmetry and feeding } \\
\text { difficulties) }\end{array}$ & $\begin{array}{l}13 \text { (extra criteria include genital } \\
\text { abnormalities; developmental } \\
\text { delay; other dysmorphic } \\
\text { features and asymmetry } \\
\text { weighted at 3-present or } \\
0 \text {-absent) }\end{array}$ & $\begin{array}{l}4 \text { Small for gestational age } \\
\text { (birth weight } S D S<-2 \text { ) } \\
\text { Postnatal short stature (after } \\
2 \text { years) (Ht SDS }<-2 \text { ) } \\
\text { Relative macrocephaly } \\
\text { (OFC }>1.5 \text { SDS than Ht SDS) } \\
\text { Asymmetry }\end{array}$ \\
\hline $\begin{array}{l}\text { Minimum } \\
\text { score for } \\
\text { clinical } \\
\text { diagnosis of } \\
\text { SRS }\end{array}$ & $3 / 5$ & $4 / 5$ & $\begin{array}{l}3 / 5 \text { plus mandatory prenatal } \\
\text { growth failure }\end{array}$ & $8 / 15$ & 3 \\
\hline
\end{tabular}

hypomethylation than in those with upd(7)mat, despite the similar postnatal growth patterns at assessment, although this was not significant (median OFC SDS H19: -0.48; upd(7)mat: -0.95 and no abnormality: $-0.95 ; \mathrm{p} 0.2$ ).

\section{Sensitivity and specificity of new scoring system}

The sensitivity $(82 \%)$ and specificity $(80 \%)$ of the proposed new scoring system are similar to the Lai et al system. ${ }^{6}$ Nine children were tested with no genetic abnormality identified (positive predictive value 67\%). In addition, four children would not have been tested but would have had a molecular genetic abnormality (negative predictive value 90.2\%). In comparison, all the currently published scoring systems had lower positive predictive values (ie, more children would have been

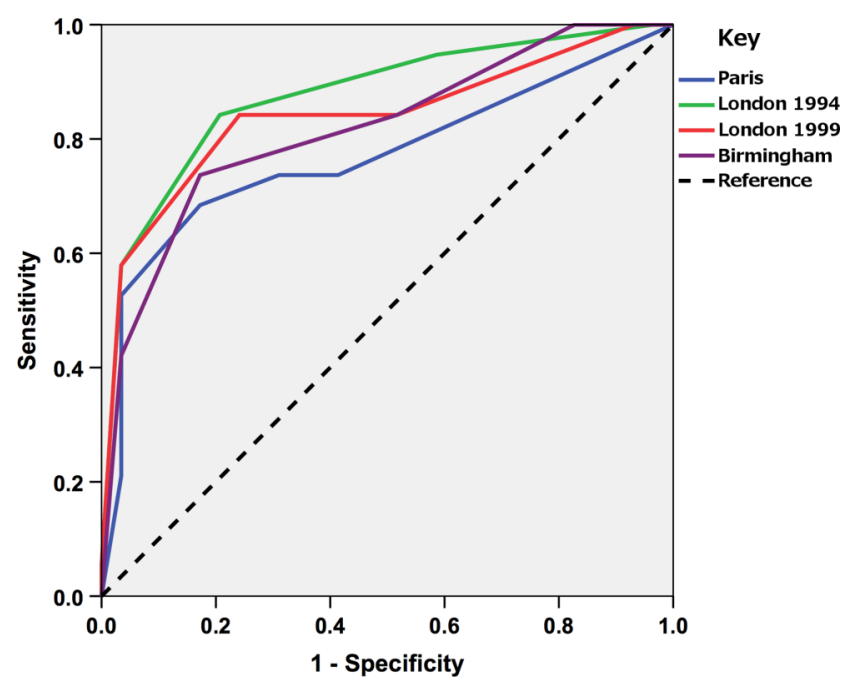

Figure 1 Receiver operator curves (ROC) for systems 1-3 compared to proposed new scoring criteria. Figure shows ROC analysis for three published scoring systems compared to proposed new scoring system as per colour key. All four perform similarly at high specificity and moderate sensitivity. tested and no genetic abnormality identified) compared to the new proposed criteria, although the negative predictive values were similar (table 2). Many children are now tested earlier than 2 years (in our cohort almost one third were referred below the age of 2 years; 33 in total). If the criteria were applied to these children, the sensitivity and specific are slightly lower but still comparable (sensitivity 78\%, specificity $75 \%$ ).

\section{DISCUSSION}

SRS is a genetically heterogeneous condition where up to $50 \%$ of all clinically diagnosed cases have no identified epi(genetic) change. It has previously been shown that patients with H19 DMR hypomethylation have a more severe growth failure phenotype and increased likelihood of asymmetry than those patients with upd(7)mat and this is seen again in our data. ${ }^{4}$ Interestingly, the differences in postnatal growth between molecular subgroups were less pronounced than either the pre-natal growth failure or other series (median height SDS: $H 19=-2.71 ; \operatorname{upd}(7) \mathrm{mat}=-2.89$ and no abnormality $=-2.32$; p 0.1 ); table 2 . $^{4}$

Previous large series of patients with SRS have been largely ascertained via research studies whereas our cohort reflected those individuals who had been referred for genetic analysis in a clinical diagnostic laboratory. We note that Turner et $a l^{16}$ found a similar fraction of patients testing positive for either upd(7) mat or hypomethylation of H19 DMR when referred to a clinical diagnostic laboratory with a potential diagnosis of SRS. Importantly, the new criteria seem to be applicable even when patients are tested below the age of 2 years (although the sensitivity and specificity are slightly lower).

A positive genetic test enables a precise diagnosis. Unfortunately, there is insufficient data on the long term clinical outcomes (such as responsiveness to growth hormone treatment or malignancy risk) in children with a clinical diagnosis of SRS according to whether they have hypomethylation of H19 DMR or upd(7)mat or no abnormality. ${ }^{17} 18$ However, although a positive genetic result may not directly affect clinical management, it does prevent further unnecessary investigation for alternative 
Table 2 Summary of scoring outcomes using the four different diagnostic criteria

\begin{tabular}{|c|c|c|c|c|c|}
\hline Scoring system & 1: Lai et $a l^{6}$ & 2: Price et $a l^{1}$ & 3: Netchine et $a l^{4}$ & 4: Bartholdi et $a l^{5}$ & 5. Birmingham \\
\hline Sensitivity (original article) & Not applicable (NA) & NA & $69 \%$ & $92 \%$ & NA \\
\hline Specificity (original article) & NA & NA & $100 \%$ & $49 \%$ & NA \\
\hline Sensitivity (Birmingham data) & $84 \%$ & $59 \%$ & $70 \%$ & Not applicable & $82 \%$ \\
\hline Specificity (Birmingham data) & $80 \%$ & $97 \%$ & $81 \%$ & Not applicable & $80 \%$ \\
\hline Negative predictive value & $95 \%$ & $80 \%$ & $91 \%$ & Not applicable & $90 \%$ \\
\hline Positive predictive value & $58 \%$ & $59 \%$ & $61 \%$ & Not applicable & $67 \%$ \\
\hline $\begin{array}{l}\text { Most common unknown } \\
\text { features }\end{array}$ & $\begin{array}{l}\text { 1. Clinodactyly }(n=28 / \\
\text { 139) } \\
\text { 2. Post-natal short } \\
\text { stature }(n=32 / 139)\end{array}$ & $\begin{array}{l}\text { 1. Relative macrocephaly } \\
\text { (OFC) }(n=59 / 139) \\
\text { 2. Post-natal short stature } \\
\text { ( } n=32 / 139\end{array}$ & $\begin{array}{l}\text { 1. OFC }(n=59 / 139) \\
\text { 2. Post-natal short } \\
\text { stature }(n=32 / 139)\end{array}$ & $\begin{array}{l}\text { 1. Low birth length ( } n=114 / 139 \text { ) } \\
\text { 2. Relative macrocephaly at birth } \\
\text { (OFC) ( } n=112 / 139)\end{array}$ & \\
\hline
\end{tabular}

OFC, occipitofrontal circumference.

causes of poor growth and removes uncertainty regarding the diagnosis for parents, so allowing them to access appropriate support groups.

As this was a study based on UK patients and retrospective, not all the data points required were available for all scoring systems, particularly for the Bartholdi assessment tool. In order to evaluate the UK based experience of genetic testing for patients with SRS, we reviewed data from an 11 year period in a supra-regional genetics testing service. Currently the UK Genetic Testing Network guidelines for clinical suspicion of SRS includes prenatal growth failure $(<0.4$ th centile) with relative macrocephaly $(>25$ th centile) and possible limb asymmetry, (http://www.ukgtn.nhs.uk/gtn/Search + for + a + Test/Search + by

+ Disease + or + Gene). These criteria are much broader than current published guidelines. We propose a new four point scoring system based on objective criteria, which is simple for one-off clinical assessment for use by a broad range of clinicians, from clinical geneticists to general paediatricians, to guide which patients should be offered molecular genetic testing in the first instance, without loss of both specificity and sensitivity.

In the Bartholdi and Netchine cohorts, the frequency of patients with H19 DMR hypomethylation ranges from 38-69\% and with upd(7)mat from 5-9\%. ${ }^{5}$ However, no evaluation of the two UK criteria (Lai and Price) has been made following the discovery of the association of SRS with H19 DMR hypomethylation. To our knowledge there have been no previous studies comparing of all four SRS scoring systems in a cohort of patients with molecular genetic testing results. As genetic testing becomes less expensive and increasingly becomes part of mainstream medical practice there is an increasing need for robust referral pathways to access molecular genetic testing in a cost effective manner, without all patients having to be seen by an expert clinician. Previously reported scoring systems for SRS have varying sensitivity and specificity, with subjective criteria that either require repeated assessment over time or previous experience of the SRS phenotype which in any case becomes more subtle in late childhood. The proposed new scoring system requires three out of four objective criteria (low birthweight, poor postnatal growth, relative macrocephaly, and asymmetry), all of which can be evaluated at a single assessment and needs no specialist training. We suggest that more sophisticated scoring systems that include subjective criteria might be more accurate in the hands of specialist clinicians with extensive experience of SRS; however, in a heterogenous group of referring clinicians (ie, outside of a research cohort), the inclusion of such variables might not improve the accuracy but might actually reduce it. Thus we note that in our referral cohort the Netchine scoring system performs less well than the simplified Birmingham criteria (table 2). For children who test negative or who do not reach the threshold for testing, but for whom a clinical diagnosis of SRS seems likely, then referral to a specialist genetics service can be undertaken for consideration of further testing (eg, if mosaicism is suspected) or alternative diagnoses (eg, microarray analysis or within a research study). An important issue for many modern health care systems is how to utilise technological advances for more accurate diagnosis while not excessively increasing healthcare expenditure. Currently commercial molecular genetic testing for SRS is between US\$400$600(£ 255-380, € 300-450)$, and it is hoped that that the new scoring system proposed here will enable a wide spectrum of clinicians to utilise this in a cost effective manner in order to facilitate earlier diagnosis of SRS.

Contributors RD, ERM were involved in study design, analysis of data and writing the article. PN and GK were involved in analysis of the data. GK was involved in collection of clinical data. CH, LT, FM were involved in molecular analysis. CH, LT, $\mathrm{SP}, \mathrm{FM}$ and TGB were involved in the final approval of the current version of the article.

Funding RPD is funded by Birmingham Children's Hospital with additional funding from Newlife Foundation and the Child Growth Foundation. ERM is funded by Action Medical Research.

Competing interests None.

Ethics approval Birmingham Women's Hospital NHS Trust Research and Development Department.

Provenance and peer review Not commissioned; externally peer reviewed.

\section{REFERENCES}

1 Price SM, Stanhope R, Garrett C, Preece MA, Trembath RC. The spectrum of Silver-Russell syndrome: a clinical and molecular genetic study and new diagnostic criteria. J Med Genet 1999:36:837-42.

2 Hitchins MP, Stanier P, Preece MA, Moore GE. Silver-Russell syndrome: a dissection of the genetic aetiology and candidate chromosomal regions. J Med Genet 2001;38:810-19.

3 Chabernaud JL, Gicquel C, Ammar F, Jourdain G, Quentin P, Castel C, Boithias C, Dehan M. Air or oxygen for neonatal resuscitation in the delivery room? I Gynecol Obstet Biol Reprod (Paris) 2005;34:S25-32.

4 Netchine I, Rossignol S, Dufourg MN, Azzi S, Rousseau A, Perin L, Houang M, Steunou V, Esteva B, Thibaud N, Demay MC, Danton F, Petriczko E, Bertrand AM, Heinrichs C, Carel JC, Loeuille GA, Pinto G, Jacquemont ML, Gicquel C, Cabrol S, Le Bouc Y. 11p15 imprinting center region 1 loss of methylation is a common and specific cause of typical Russell-Silver syndrome: clinical scoring system and epigenetic-phenotypic correlations. J Clin Endocrinol Metab 2007;92:3148-54.

5 Bartholdi D, Krajewska-Walasek M, Ounap K, Gaspar H, Chrzanowska KH, llyana H, Kayserili $H$, Lurie IW, Schinzel A, Baumer A. Epigenetic mutations of the imprinted IGF2-H19 domain in Silver-Russell syndrome (SRS): results from a large cohort of patients with SRS and SRS-like phenotypes. J Med Genet 2009;46:192-7. 
6 Lai KY, Skuse D, Stanhope R, Hindmarsh P. Cognitive abilities associated with the Silver-Russell syndrome. Arch Dis Child 1994;71:490-6.

7 Scott RH, Douglas J, Baskcomb L, Nygren AO, Birch JM, Cole TR, Cormier-Daire V, Eastwood DM, Garcia-Minaur S, Lupunzina P, Tatton-Brown K, Bliek J, Maher ER, Rahman N. Methylation-specific multiplex ligation-dependent probe amplification (MS-MLPA) robustly detects and distinguishes $11 \mathrm{p} 15$ abnormalities associated with overgrowth and growth retardation. J Med Genet 2008;45:106-13.

8 Priolo M, Sparago A, Mammi C, Cerrato F, Lagana C, Riccio A. MS-MLPA is a specific and sensitive technique for detecting all chromosome 11 p15.5 imprinting defects of BWS and SRS in a single-tube experiment. Eur J Hum Genet 2008;16:565-71.

9 Wakeling EL, Amero SA, Alders M, Bliek J, Forsythe E, Kumar S, Lim DH, MacDonald F, Mackay DJ, Maher ER, Moore GE, Poole RL, Price SM, Tangeraas T, Turner CL, Van Haelst MM, Willoughby C, Temple IK, Cobben JM. Epigenotype-phenotype correlations in Silver-Russell syndrome. J Med Genet 2010;47:760-8.

10 Halliday J, Oke K, Breheny S, Algar E, D JA. Beckwith-Wiedemann syndrome and IVF: a case-control study. Am J Hum Genet 2004;75:526-8.

11 Maher ER, Afnan M, Barratt CL. Epigenetic risks related to assisted reproductive technologies: epigenetics, imprinting, ART and icebergs? Hum Reprod 2003;18:2508-11.

12 Maher ER, Brueton LA, Bowdin SC, Luharia A, Cooper W, Cole TR, Macdonald F, Sampson JR, Barratt CL, Reik W, Hawkins MM. Beckwith-Wiedemann syndrome and assisted reproduction technology (ART). J Med Genet 2003;40:62-4.
13 Lim D, Bowdin SC, Tee L, Kirby GA, Blair E, Fryer A, Lam W, Oley C, Cole T, Brueton LA, Reik W, Macdonald F, Maher ER. Clinical and molecular genetic features of Beckwith-Wiedemann syndrome associated with assisted reproductive technologies. Hum Reprod 2009;24:741-7.

14 Kagami M, Nagai T, Fukami M, Yamazawa K, Ogata T. Silver-Russell syndrome in a girl born after in vitro fertilization: partial hypermethylation at the differentially methylated region of PEG1/MEST. J Assist Reprod Genet 2007;24:131-6.

15 Ferraretti AP, Goossens V, de Mouzon J, Bhattacharya S, Castilla JA, Korsak V, Kupka M, Nygren KG, Nyboe Andersen A. Assisted reproductive technology in Europe, 2008: results generated from European registers by ESHRE. Hum Reprod 2012;27:2571-84.

16 Turner CL, Mackay DM, Callaway JL, Docherty LE, Poole RL, Bullman H, Lever M, Castle BM, Kivuva EC, Turnpenny PD, Mehta SG, Mansour S, Wakeling EL, Mathew V, Madden J, Davies JH, Temple IK. Methylation analysis of 79 patients with growth restriction reveals novel patterns of methylation change at imprinted loci. Eur J Hum Genet 2010;18:648-55.

17 Rakover Y, Dietsch S, Ambler GR, Chock C, Thomsett M, Cowell CT. Growth hormone therapy in Silver Russell syndrome: 5 years experience of the Australian and New Zealand Growth database (OZGROW). Eur J Pediatr 1996;155:851-7.

18 Chatelain PG. Auxology and response to growth hormone treatment of patients with intrauterine growth retardation or Silver-Russell syndrome: analysis of data from the Kabi Pharmacia International Growth Study. International Board of the Kabi Pharmacia International Growth Study. Acta Paediatr Supp/ 1993;82(Supp 391):79-81. 